\title{
Transfer Hydrogenation Catalyzed by Organometallic Complexes using NADH as Reductant in a Biochemical Context
}

\author{
Yasunori Okamoto, Thomas R. Ward* \\ Department of Chemistry, University of Basel, Mattenstrasse 24a, BPR 1096, CH-4058 Basel, Switzerland
}

Transfer hydrogenation of $\mathrm{C}=\mathrm{O}$ and $\mathrm{C}=\mathrm{N}$ bonds is one of the cornerstones of synthetic organic methodology. In this context and thanks to their remarkable stability against air and water, $\mathrm{d}^{6}$-piano stool complexes of $\mathrm{Ru}$, $\mathrm{Rh}$, and Ir have found wide applications as homogeneous catalysts both in organic solvents and in aqueous media. Most frequently, either formic acid or 2-propanol are used as hydride source.

Recently, the $d^{6}$-piano stool-catalyzed transfer hydrogenation has found its way in a biological context for the regeneration of the $\mathrm{NAD}(\mathrm{P})^{+} / \mathrm{NAD}(\mathrm{P}) \mathrm{H}$ couple. Indeed and in view of its use as cofactor in over 400 redox enzymes, the non-enzymatic regeneration of NADH by $\mathrm{d}^{6}-$ pianstool complexes has attracted significant interest in the past thirty years. In a biomimetic spirit, the use of $\mathrm{NADH}$ as hydride source for the generation a $\left\{\mathrm{Cp}^{*} \mathrm{M}-\right.$ $\mathrm{H}\}$-moiety $\left(\mathrm{Cp}^{*}=\eta^{5}-\mathrm{C}_{5} \mathrm{Me}_{5}{ }^{-}\right)$has been also achieved. In stark contrast to enzymes, homogeneous catalysts very rarely can catalyze both the forward and the reverse transformations. Careful design of $\mathrm{d}^{6}$-piano stool complexes now allows to use NADH for the reduction of dioxygen, quinones, imines and aldehydes both in vitro and in vivo (Scheme 1).

Transfer hydrogenation using intracellular NADH was utilized to promote anticancer activity. The group of Sadler demonstrated that Ir A (Scheme 1a) catalyzes the transfer hydrogenation of molecular oxygen to produce hydrogen peroxide using NADH as reductant (reaction i, Scheme 1b). ${ }^{1}$ Hydrogen peroxide is one of the reactive oxygen species (ROS), which causes oxidative stress. The Ir A complex catalyzed the production of hydrogen peroxide, thus displaying anticancer activity, which proved to be higher than that of cisplatin.

In a related context, the group of Komatsu and Ariga demonstrated intracellular NADH imaging relying on its use as hydride source to quench the fluorescence of a reporter probe. They showed that Ir B (Scheme 1a) could use NADH as hydride source for the transfer hydrogenation of an ubiquinone moiety in a rhodol derivative (UQ-Rh 1) (reaction ii, Scheme 1b). ${ }^{2}$ The fluorescence of UQ-Rh 1 decreases upon reduction of the UQ moiety. Addition of Ir $\mathbf{B}$ to Hela cells treated by UQ-Rh $\mathbf{1}$ resulted in fluorescence decrease, indicating that $\operatorname{Ir} \mathbf{B}$ is able to catalyze transfer hydrogenation using NADH in the Hela cells. Accordingly, imaging of NADH was successfully demonstrated, albeit relying on a turn-off fluorescent probe.

With synthetic biology applications in mind, transfer hydrogenation was implemented to multienzymatic cascades. The group of Ward constructed an NAD(P)Hdependent artificial transfer hydrogenase (ATHase) by incorporation of Ir $\mathbf{C}$ into streptavidin relying on the biotin-(strept)avidin technology (Scheme 1a). ${ }^{3}$ This artificial/natural enzymatic cascades performed asymmetric imine reduction and worked at intracellular NAD $(\mathrm{P}) \mathrm{H}$ concentrations (reaction iii, Scheme 1b).

Despite the few examples highlighted above, the use of organometallic complexes as catalysts in vivo remains challenging. This can be traced back to the numerous metabolites and enzymes that may bind with high affinity to $\mathrm{d}^{6}$-piano stool complexes, thus inhibiting catalysis. To overcome this challenge, the group of Do screened and identified organometallic complexes that are stable in the presence of various biomolecules and can use NADH as hydride source. They selected the reduction of aldehydes as catalytic transformation. Indeed, aldehydes derived from lipid peroxidation have been implicated in various diseases: reduction of these aldehydes to the corresponding alcohols may lead to detoxification. Screening of bidentate ligands, lead to the identification of complex Ir D (Scheme 1a) that could reduce cytotoxic aldehydes with NADH (reaction iv, Scheme 1b). ${ }^{4}$ Gratifyingly, Ir $\mathbf{D}$ remained active in cell culture media and even in the presence of biological nucleophiles, highlighting the high tolerance of Ir $\mathbf{D}$ to physiological conditions.

This achievement prompted them to test Ir $\mathbf{D}$ for intracellular transfer hydrogenation. ${ }^{5}$ As reporter, they selected a turn-on fluorescent probe. They prepared various fluorophores bearing an aldehyde moiety as substrate for transfer hydrogenation. Among substrates tested, Bodipy-modified with an aldehyde group (Bodipy-CHO 7) was identified as a suitable substrate. Its reduced form, Bodipy-OH 8, displays a fivefold higher emission than Bodipy-CHO 7. For the in vitro experiment, a twofold increase in fluorescence emission was 
observed after $4 \mathrm{~h}$ upon reduction of Bodipy-CHO 7 (reaction v, Scheme 1b).

Next, they tested transfer hydrogenation of Bodipy$\mathrm{CHO} 7$ in vivo. For this purpose, the NIH-3T3 mouse embryo fibroblast cells were selected. After incubation with Bodipy-CHO 7, cells were washed and treated with Ir D. Compared with a control experiment lacking Ir D, a 1.6-fold higher fluorescence of the cells was observed after $2 \mathrm{~h}$. This result suggests that transfer hydrogenation by Ir $\mathbf{D}$ occurs thanks to the presence of intracellular NADH as a hydride source. Ir $\mathbf{D}$ also catalyzes this reaction in A549 human epithelial cancer cells. Importantly, the intracellular concentration of Ir $\mathbf{D}$ was quantified by ICP-MS.

To ensure that intracellular NADH is indeed the hydride source they modulated the intracellular NADH level in cells. Addition of sodium pyruvate, a natural metabolite that signals the slowdown of glycolysis leading to lower NADH levels, resulted in lower fluorescence caused by Bodipy-OH 8.

As Do et al. demonstrated, despite the marked affinity of soft precious metals for various metabolites, most notably thiols and thioethers, careful identification of suitable bidentate ligands for the $\{\mathrm{Cp} * \operatorname{Ir}\}$-moiety, allows to carry out transfer hydrogenation in vivo using $\mathrm{NADH}$ as hydride source. These findings open fascinating perspectives for the catalytic uncaging of drugs by organometallic complexes in vivo via transfer hydrogenation. To achieve this feat however, further efforts are required to improve the catalytic performance (i.e. turnover numbers and saturation kinetic parameters).

Scheme 1. Various $d^{6}$-pianostool iridium complexes (a) catalyze transfer hydrogenation reactions using NADH as hydride source (b). a)

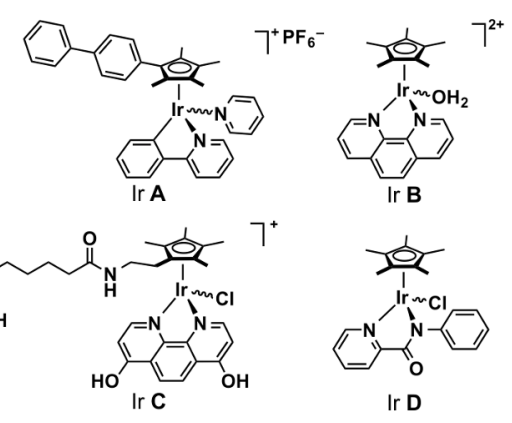

b)

(i)

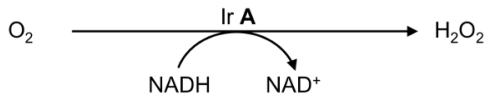

(ii)
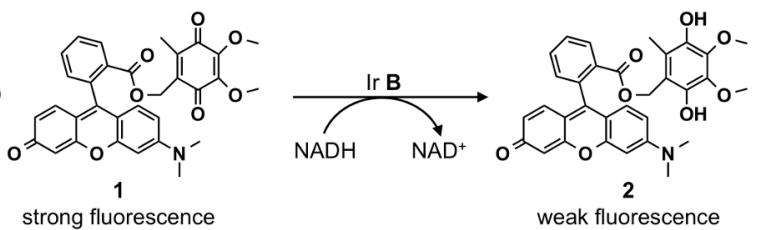

(iii)

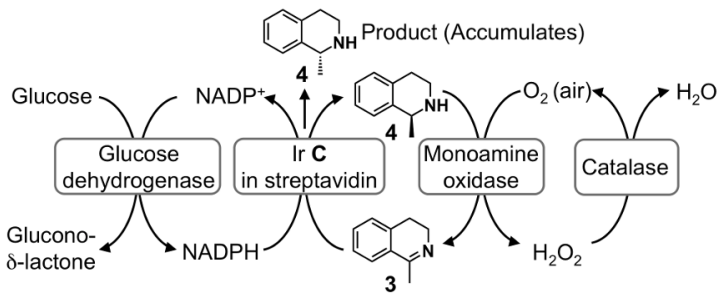

(iv)
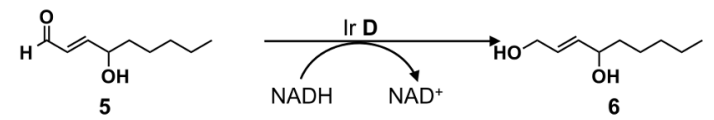

(v)
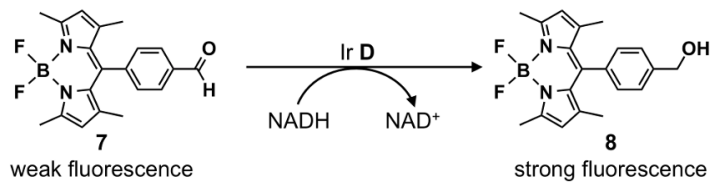

\section{AUTHOR INFORMATION}

\section{Corresponding Author}

*E-mail: thomas.ward@unibas.ch

Note

The authors declare no competing financial interest.

\section{REFERENCES}

(1)

Liu, Z.; Romero-Canelón, I.; Qamar, B.; Hearn, J. M.; Habtemariam, A.; Barry, N. P. E.; Pizarro, A. M.; Clarkson, G. J.; Sadler, P. J. (2014) The potent oxidant anticancer activity of organoiridium catalysts. Angew. Chem., Int. Ed. 53, 3941-3946.

(2)
Komatsu, H.; Shindo, Y.; Oka, K.; Hill, J. P.; Ariga, K. (2014) Ubiquinone-rhodol (UQ-Rh) for fluorescence imaging of $\mathrm{NAD}(\mathrm{P}) \mathrm{H}$ through intracellular activation. Angew. Chem., Int. Ed. 53, 3993-3995. 
(3) Okamoto, Y.; Köhler, V.; Ward, T. R. (2016) An NAD(P)Hdependent artificial transfer hydrogenase for multienzymatic cascades. J. Am. Chem. Soc. 138, 5781-5784.

(4) genation of cytotoxic aldehydes using nicotinamide adenine dinucleotide (NADH) in cell growth media. ACS Catal. 6 , 2637-2641.

(5) Bose, S.; Ngo, A. H.; Do, L. H. (2017) Intracellular transfer hydrogenation mediated by unprotected organoiridium catalysts. J. Am. Chem. Soc. 139, 8792-8795. 
Authors are required to submit a graphic entry for the Table of Contents (TOC) that, in conjunction with the manuscript title, should give the reader a representative idea of one of the following: A key structure, reaction, equation, concept, or theorem, etc., that is discussed in the manuscript. Consult the journal's Instructions for Authors for TOC graphic specifications.

\section{Insert Table of Contents artwork here}

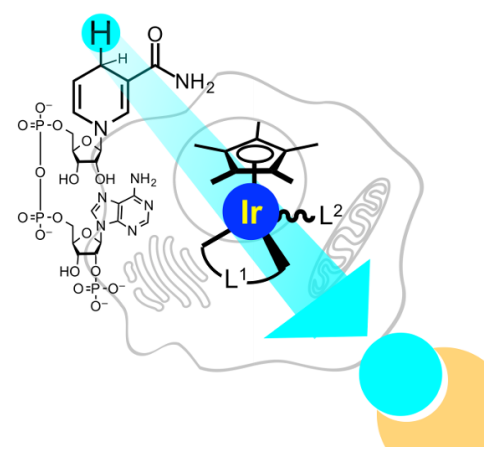

